\section{Clostridium perfringens liver abscess complicated by bacteremia}

A 63-year-old man had previously undergone resection for stage III colon cancer. Liver metastasis had resolved with palliative chemotherapy. The patient was now admitted because of weight loss, obstructive jaundice, and intrahepatic and extrahepatic biliary dilatation due to extrinsic compression of the biliary tree at the porta hepatis.

Endoscopic retrograde cholangiography (ERCP) was performed with placement of a fully covered metal stent in the common bile duct and right hepatic duct, and the patient was discharged with downward-trending bilirubin levels. However, 10 days later, he was readmitted with dyspnea, abdominal pain, and a temperature of $100.1^{\circ} \mathrm{F}$.

Physical examination revealed chest pain at the left lower ribs and epigastric tenderness. Laboratory test results were white blood cell count $21.3 \times 10^{9} / \mathrm{L}$, bilirubin $7.6 \mathrm{mg} / \mathrm{dL}$, alkaline phosphatase $212 \mathrm{U} / \mathrm{L}$, and blood cultures positive for Clostridium perfringens ( $\bullet$ Fig. 1 ). Computed tomography of the abdomen showed a $3.1-\mathrm{cm}$ collection of fluid that contained gas in the left lobe of the liver ( $\bullet$ Fig. 2 ). Inadequate drainage of the left hepatic system due to blockage by the stent in the right hepatic duct may have created a nidus for infection. The patient was successfully treated with intravenous antibiotics, abscess drainage, and removal of the metal stent ( $\bullet$ Fig.3).

C. perfringens is a rapidly growing (doubling time of 7 minutes), spore-forming, anaerobic, Gram-positive bacillus that is often found in the human bowel but rarely causes liver abscess and sepsis. To the best of our knowledge, approximately 22 cases of $C$. perfringens liver abscess resulting in septicemia have been reported, many of which were fatal [1]. The infections that lead to septicemia after ERCP are most commonly caused by Escherichia coli, Pseudomonas aeruginosa, and Klebsiella pneumoniae, but rarely $C$. perfringens [1] C. perfringens infection has a mortality rate of $70 \%$ to $100 \%$, often develops in immunosuppressed patients, and presents with fever, abscess, or hemolysis [2]. Liver abscess due to $C$. perfringens is a rare and often fatal event; a high degree of suspicion for the diagnosis and immediate treatment with drainage and antibiotics are required.

\section{Endoscopy_UCTN_Code_CPL_1AK_2AC}

Competing interests: None

\section{Charles Rives ${ }^{1}$, Dhara Chaudhari ${ }^{2}$, James Swenson², Chakradhar Reddy², Mark Young ${ }^{2}$}

${ }^{1}$ Department of Internal Medicine,

East Tennessee State University James

H. Quillen College of Medicine, Johnson City, Tennessee, USA

2 Department of Gastroenterology, East Tennessee State University James $\mathrm{H}$. Quillen College of Medicine, Johnson City, Tennessee, USA

\section{References}

1 Shindo Y, Dobashi Y, Sakai T et al. Epidemiological and pathobiological profiles of Clostridium perfringens infections: review of consecutive series of 33 cases over a 13-year period. Int J Clin Exp Pathol 2015; 8: 569 577

2 Paulino C, Silvestre J, Goncalves PereiraJ et al. Clostridium perfringens sepsis with massive intravascular haemolysis: a rare presentation. J Medical Cases 2012; 3: 207-210

\section{Bibliography}

DOI http://dx.doi.org/

10.1055/s-0034-1392867

Endoscopy 2015; 47: E457

(c) Georg Thieme Verlag KG

Stuttgart · New York

ISSN 0013-726X

\section{Corresponding author}

\section{Dhara Chaudhari, MD}

Department of Gastroenterology

East Tennessee State University

1008 Quality Circle

Apartment No. 79

Johnson City, TN 37615

USA

Fax: +1-423-741-9292

Chaudharidhara@yahoo.com

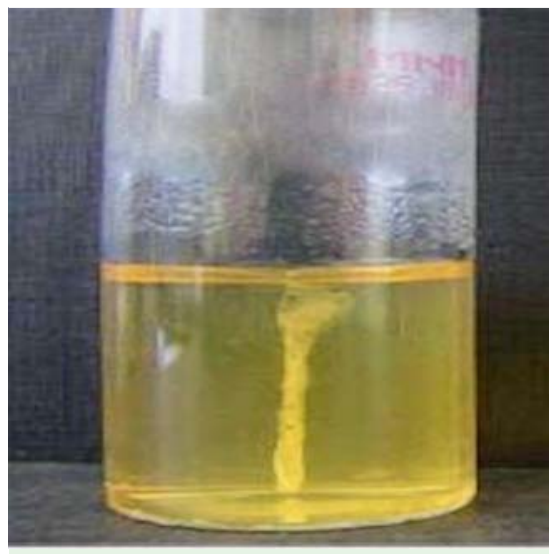

Fig. 1 Culture bottle showing gas bubbles in the direction of a swab stick from a 63-year-old patient presenting with dyspnea, abdominal pain, and a temperature of $100.1^{\circ} \mathrm{F}$ following placement of a fully covered metal stent in the common bile duct and right hepatic duct.

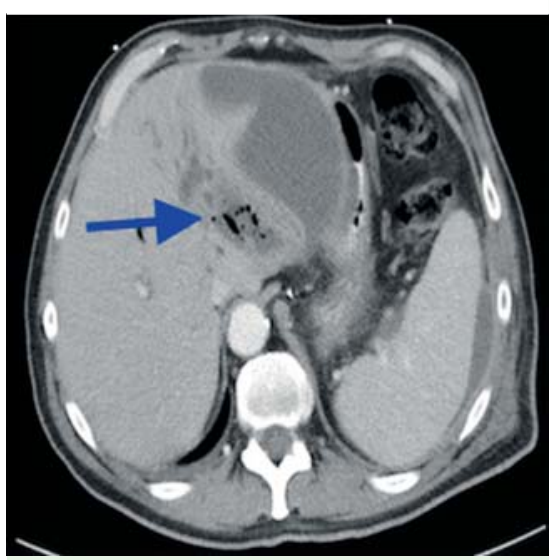

Fig. 2 Computed tomographic scan of the abdomen showing a liver abscess (arrow) containing gas.

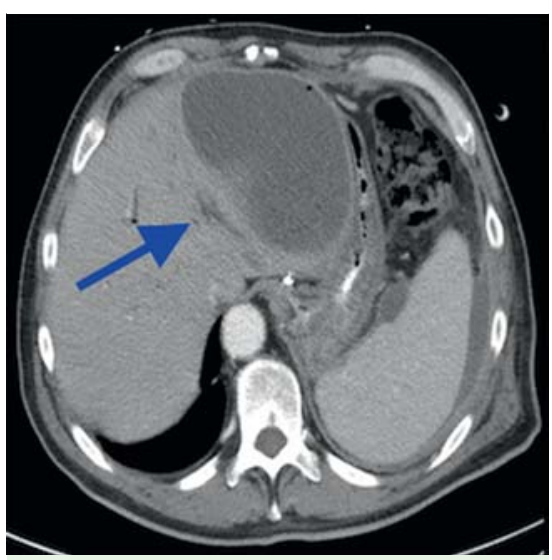

Fig. 3 Computed tomographic scan of the abdomen showing regression of the liver abscess (arrow) after drainage and antibiotic therapy for 3 weeks. 